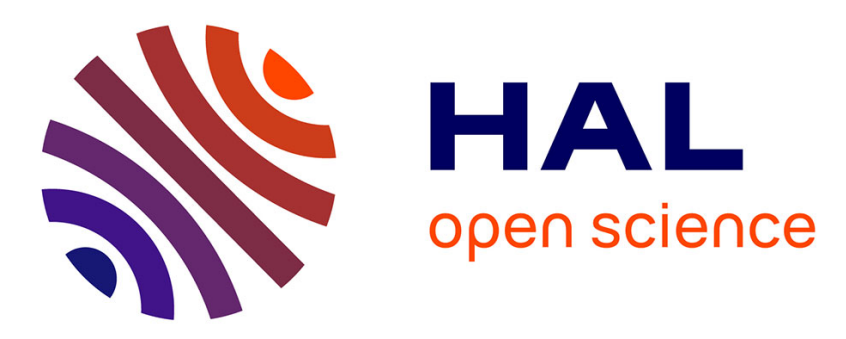

\title{
Stochastic modelling and prediction of fatigue crack propagation using piecewise-deterministic Markov processes
}

Anis Ben Abdessalem, Romain Azaïs, M Touzet-Cortina, A Gégout-Petit, M Puiggali

\section{To cite this version:}

Anis Ben Abdessalem, Romain Azaïs, M Touzet-Cortina, A Gégout-Petit, M Puiggali. Stochastic modelling and prediction of fatigue crack propagation using piecewise-deterministic Markov processes. 2015. hal-01178988v2

\section{HAL Id: hal-01178988 \\ https://hal.science/hal-01178988v2}

Preprint submitted on 30 Apr 2016

HAL is a multi-disciplinary open access archive for the deposit and dissemination of scientific research documents, whether they are published or not. The documents may come from teaching and research institutions in France or abroad, or from public or private research centers.
L'archive ouverte pluridisciplinaire HAL, est destinée au dépôt et à la diffusion de documents scientifiques de niveau recherche, publiés ou non, émanant des établissements d'enseignement et de recherche français ou étrangers, des laboratoires publics ou privés. 


\title{
Stochastic modelling and prediction of fatigue crack propagation using piecewise-deterministic Markov processes
}

\author{
A. Ben Abdessalem ${ }^{\S}$, R. Azaïs ${ }^{\dagger, \natural}$, M. Touzet-Cortina*‡, A. Gégout-Petitt, ${ }^{\dagger, \natural}$ and M. Puiggali ${ }^{\S}$ \\ §Université de Bordeaux, I2M CNRS UMR 5295, France \\ †Inria Nancy-Grand Est, Team BIGS \\ ${ }^{\natural}$ Institut Élie Cartan de Lorraine, Université de Lorraine, Nancy, France \\ ${ }^{\ddagger}$ Bordeaux INP, I2M CNRS UMR 5295, France
}

\begin{abstract}
Fatigue crack propagation is a stochastic phenomenon due to the inherent uncertainties originating from material properties, environmental conditions and cyclic mechanical loads. Stochastic processes offer thus an appropriate framework for modelling and predicting crack propagation. In this paper, the fatigue crack growth is modelled and predicted by a piecewise-deterministic Markov process associated with deterministic crack laws. First, a regime-switching model is used to express the transition between Paris' regime and rapid propagation which occurs before failure. Both regimes of propagation are governed by a deterministic equation whose parameters are randomly selected in a finite state space. This one has been adjusted from real data available in the literature. The crack growth behaviour is well-captured and the transition between both regimes is well-estimated by a critical stress intensity factor range. The second purpose of our investigation deals with the prediction of the fatigue crack path and its variability based on measurements taken at the beginning of the propagation. The results show that our method based on this class of stochastic models associated with an updating method provides a reliable prediction and can be an efficient tool for safety analysis of structures in a large variety of engineering applications. In addition, the proposed strategy requires only few information to be effective and is not time-consuming.
\end{abstract}

Keywords: Fatigue crack propagation, Uncertainties, Stochastic processes, Piecewise-deterministic Markov processes, Regime-switching models, Prediction

\section{Introduction}

Fatigue crack growth (FCG) in materials exhibits a wide range of scatter even under controlled experimental conditions, see $[1,2,3,4,5]$. Various sources of scatter exist during the fatigue life, which can be divided into crack initiation and crack growth periods. For each period, the sources of uncertainties may be different. It is well-known that crack initiation, including the first micro-crack growth is a discontinuous process which is dependent on mechanical parameters but also on microstructure and material surface quality (local surface inhomogeneities, small surface irregularities and slight surface damage). In the second period, the crack growth mainly depends on mechanical parameters (applied loads, mechanical properties, etc.). For these reasons, it is technically significant to consider crack initiation and crack growth periods separately. In the present paper, the work is only focused on the crack growth periods.

Stochastic modelling strategies are relevant to consider the effects of uncertainties. Some papers have addressed the stochastic process framework to model fatigue crack propagation. Indeed, this context enables

\footnotetext{
${ }^{*}$ Corresponding author; e-mail: marie-benedicte.touzet-cortina@u-bordeaux.fr
} 
the introduction of certain variabilities to the typical deterministic laws to describe FCG under constant or variable amplitude fatigue loading, see for instance $[6,7,8,9,10,11,12,13]$ and the references therein. Among the deterministic models of FCG proposed in the literature, the Paris-Erdogan and Forman laws, respectively proposed in [14] and [15], are widely used because of their simplicity and the limited number of parameters involved. Certain strategies are provided to input randomness into these models and to treat FCG from a stochastic point of view. A popular idea suggested in the literature consists in adding to the deterministic law a multiplicative noise function which is generally a non-negative random process, see for instance $[3,16]$. For example, Gaussian white noise is used in [17] to add randomness to the deterministic FCG law. Coupled with a filtering technique, this modified law excludes the possibility of negative crack growth rates. The same strategy is followed in [18]. The authors model the noise function by a stationary lognormal random process with median unity. Another possibility to put randomness in the model is to consider the parameter of the FCG as random variables [19]. The strip-yield model included in the NASGRO software developed in [20] is widely used to simulate crack growth under variable amplitude loading, see for instance [21]. More recently, the authors of [22] used the polynomial chaos expansions in combination with the Karhunen-Loève series for accurate and efficient representation of random crack propagation data. They used two different experimental data sets from literature to demonstrate the ability of their model to simulate and predict the fatigue crack propagation.

Markov processes are also proposed to address stochastic modelling of FCG. In [23], the authors used Markov chains to propagate the variability affecting non-proportional load sequences. The authors of [24] considered discrete Markov methods to handle the uncertainties of initial crack length and loading. A model based on the fracture mechanics theory and the diffusive Markov processes is derived in [25] to treat the variabilities affecting material resistance and stress loading. Among the Markov processes suitable to perform crack modelling, one may also consider the class of piecewise-deterministic Markov processes (PDMP's) frequently employed in safety and reliability. These stochastic models have been introduced in [26] to handle both discrete events (changes of regime or failures in our context) and continuous evolution of physical phenomena (crack length in our context). To the best of our knowledge, [27] was the first paper to use PDMP's to model FCG as a degradation mechanism that continuously evolves in time with the growth rate changing at random times. Among the papers about stochastic models cited above, $[16,19,7,8,10,11]$ propose prediction and validation methods. The reader can also refer to [28] in which the authors propose an inference method based on maximum relative entropy in order to predict the propagation phase of a crack knowing only few points of the beginning of the propagation.

Even if among the aforementioned works, the effects of random loading have been sometimes investigated, the study is limited to the source of uncertainties related to material properties observed in large replicate fatigue crack propagation tests. Therefore, we use in this article the data set presented in [1] and composed of 62 specimens of 2024-T3 aluminium alloys tested under constant amplitude loading. The aim of this paper is to show the ability of PDMP's to model crack propagation in order to tackle two specific problems: the first one is to capture the transition time between two regimes of propagation and the second one is to predict the behaviour of a crack until the exit of the linear Paris regime using the first experimental points of its propagation as conditional events.

The objective of the first part of this work is to detect the conditions of crack growth instability that is the transition between the stable region of propagation and the unstable one when the crack growth extends in a rapid manner. In this way, a regime-switching model is proposed: two deterministic laws are combined using the more suitable one for each specific period. In the second part, the model is associated with an updating method to predict the whole trajectory of a given crack in order to manage the structure safety using experimental data provided by non-destructive control. The potential application of the method is to give a prediction of the number of loading cycles that allows the crack to reach a critical length. It might be used to guarantee safety for a mission if the predicted number of cycles is widely greater than the one required for the mission. FCG is modelled by a PDMP whose deterministic flow is given by either Paris-Erdogan or Forman law. Uncertainties are integrated through the material parameters of the above-mentioned laws and through the transition time between regimes of propagation. 
The layout of the paper is as follows. In Section 2, the model formulation is exposed via PDMP's and based on known mechanistic crack growth laws. Section 3 is dedicated to the transition between the two regions of propagation through our model. Section 4 deals with the prediction of crack propagation using PDMP's associated with an updating method. Finally, Section 5 gives some elements of discussion and perspectives.

\section{Model formulation}

\subsection{Definition of PDMP's}

Piecewise-deterministic Markov processes form a class of non-diffusion stochastic models introduced in the literature in [26] in operational research to model physical systems whose dynamics can be disrupted by punctual and random events (non-continuous changes). Consequently, PDMP's are described by two variables: a usual Euclidean state and a discrete variable (called mode or regime) which takes values in a discrete set. In this context, the state variable is representative of the physical system (state, speed, length of a crack, etc.) while the discrete variable reflects the operating mode or a regime of evolution (region of propagation in our case). It should be noted that the dynamic of the Euclidean variable of a PDMP is governed, between two jumps, by a deterministic differential equation, unlike models often encountered in the literature in which the physical states are assumed to be piecewise-constant or diffusive. The randomness of such a process comes from the jump times and the punctual changes occuring at these times.

More precisely, a PDMP is a two-component process $\left(\mu_{t}, X_{t}\right)$ where the mode $\mu_{t}$ takes its values in a finite state space $\mathcal{M}$, while $X_{t}$ represents the physical variable and evolves in an open subset of $\mathbb{R}$ or $\mathbb{R}^{d}$. The motion of the continuous part $X_{t}$ is governed by the flow $\mathcal{G}$ of a deterministic differential equation whose parameters are directed by the mode $\mu_{t}$. The trajectory of such a process is defined in a very intuitive and iterative way: starting from $\left(\mu_{0}, X_{0}\right)=(M, x)$, the path is given between times 0 and $T_{1}$ by:

$$
\mu_{t}=M \quad \text { and } \quad X_{t}=\mathcal{G}_{M}(x, t),
$$

where $T_{1}$ is the first jump time whose distribution depends on the couple $(M, x)$. At time $T_{1}$, a jump occurs according to a jump rate, and the mode is switched according to a transition matrix that gives the probability from one state to another, and so on. Note that even if the PDMP framework allows at time $T_{1}$ a jump of the continuous physical variable $X_{t}$ of the model, in most of the applications the jump occurs only on the discrete part $\mu_{t}$ of the process. In our case, a jump at time $T_{1}$ only affects the discrete variable $\mu_{t}$. The following diagram is given to sum up the procedure:

- The process starts from $\left(\mu_{0}, X_{0}\right)=(M, x)$;

- The first jump time $T_{1}$ is a random function of $(M, x)$;

- For any time $0 \leq t<T_{1}, \mu_{t}=M$ and $X_{t}$ is a deterministic function of $M$ and $x$, that is $X_{t}=\mathcal{G}_{M}(x, t)$;

- At time $T_{1}, X_{T_{1}}=\mathcal{G}_{M}\left(x, T_{1}\right)$ and $\mu_{T_{1}}$ is a random function of $M$ and $X_{T_{1}}$ whose distribution is given by a transition matrix;

- Back to first step from $\left(\mu_{T_{1}}, X_{T_{1}}\right)$.

An appropriate selection of the state space and of the main features of the process provide a large variety of stochastic models covering many applications as management of complex systems (see [29, 30]), modelling of degradation (see [31]) or biology (see [32]). The mathematical properties and the numerical implementations of these processes are the main topic of many recent publications, see [26, 33, 34, 35, 36, 37] and the references therein. In the present paper, the selected stochastic models involve differential equations between jumps such as Paris-Erdogan and Forman laws described in the sequel. 


\subsection{Deterministic crack growth models}

For ductile materials, FCG rate can be correlated with the cyclic variation of stress intensity factor. The typical logarithmic plot of $\mathrm{d} a / \mathrm{d} N$ versus $\Delta K$ is shown in Figure 1: the curve is divided into 3 regions. In region I, referred to near-threshold region, crack propagation is a discontinuous process which is extremely slow at very lows values of $\Delta K$. In the large and linear region II, a power-law relationship between crack growth rate and stress intensity factor range is observed. Finally, region III corresponds to the increase of crack propagation rate when the stress intensity factor tends to the critical value $K_{C}$. Many deterministic laws have been proposed in the literature for modelling the crack behaviour in region II. Among them, Paris-Erdogan's law is certainly the most used for steels and aluminium alloys. It was introduced in [14] and is defined from the following equation:

$$
\frac{\mathrm{d} a}{\mathrm{~d} N}=C(\Delta K)^{m}
$$

where $\mathrm{d} a / \mathrm{d} N$ is the crack growth rate per cycle, $a$ is the fatigue crack length, $N$ is the number of cycles, $C$ and $m$ are the Paris' law parameters and $\Delta K$ is the range of the stress intensity factor. In most cases, $\Delta K$ is given by the formula:

$$
\Delta K=Y(a) \Delta \sigma \sqrt{\pi a},
$$

where $Y(a)$ is a dimensionless factor that considers the crack shape and the geometry of the specimen and $\Delta \sigma=\sigma_{\max }-\sigma_{\min }$ is the stress range.

Even with its popularity and its accuracy in describing the region II of propagation, the model given by equation (1) is not well-adapted to express the transition at the beginning of region III. Among the variants of Paris equation developed to overcome this drawback, the authors of [15] suggested a model called Forman law and given by equation (3). This law captures the rapid increasing of growth in region III and includes the stress ratio $R=\sigma_{\min } / \sigma_{\max }$ and the fracture toughness $K_{C}$ :

$$
\frac{\mathrm{d} a}{\mathrm{~d} N}=\frac{C(\Delta K)^{m}}{(1-R) K_{C}-\Delta K},
$$

where $K_{C}$ represents the maximal value of the stress intensity factor required to induce failure. In our approach, the fracture toughness $K_{C}$ is assumed to be fixed and known.

Figure 1 approximately here.

\subsection{PDMP's applied to fatigue crack propagation}

PDMP's are suitable for modelling and predicting degradation processes induced by the presence of cracks in structural components. In particular, the changes of regime at jump times are suitable to express the damage induced by crack propagation. Within this context, the authors of [27] consider that FCG changes through small shocks occurring at random times. Precisely, these authors choose to use PDMP's without any constraint on the number of possible jumps associated with the deterministic Paris' law. They note that the jump rate is not time-homogeneous since the frequency of jumps increases at the end of the path. Unlike this approach, it is assumed that crack propagation can be expressed by a simple PDMP with only one change of regime (or jump) in order to give a physical meaning to this jump and also to bring some flexibility to the model called regime-switching model. In the first case presented in Section 3, the jump can express the transition between two regimes of propagation when two distinct laws are used before and after the jump. In this case, the deterministic flow $\mathcal{G}$ driving the propagation between two jumps is given by either the differential equation (1) in the first part or equation (3) in the second part. In Section 4, this idea may also give some flexibility to a model of propagation based on a unique law. Indeed, for the model presented in Section 4, the differential equation $\mathcal{G}$ driving the propagation will be given by equation (1) but its parameters will change at the jump time. The main ideas for the construction of the model are given in the sequel. 
- The equation driving the evolution of the crack length before the change of regime $\left(\mathcal{G}_{M_{1}}\right)$ or after $\left(\mathcal{G}_{M_{2}}\right)$ is chosen between Paris law (1) and Forman law (3). Both equations depend on a two-dimensional parameter $(m, C)$.

- At time $0,\left(m_{1}, C_{1}\right)$ is randomly selected in a finite state space $\mathcal{M}$, and FCG deterministically evolves according to the deterministic equation of propagation given $\mathcal{G}_{M_{1}}$ with $(m, C)=\left(m_{1}, C_{1}\right)$ until a random time denoted by $T$. Next, new parameters $\left(m_{2}, C_{2}\right)$ are selected according to a Markov transition on $\mathcal{M}$ : after $T$, the process follows the equation $\mathcal{G}_{M_{2}}$ with parameters $\left(m_{2}, C_{2}\right)$.

The model is thus defined from the following features:

- The two equations of propagation $\mathcal{G}_{M_{1}}$ and $\mathcal{G}_{M_{2}}$. It should be noted that they can be obtained from the same mechanistic law.

- The initial distribution of $\left(m_{1}, C_{1}\right)$, the parameters of $\mathcal{G}_{M_{1}}$, given by a probability distribution on $\mathcal{M}$.

- The law of the jump time depending on the parameters $\left(m_{1}, C_{1}\right)$.

- The law of transition between the parameters of the first regime $\left(m_{1}, C_{1}\right)$ and the second one $\left(m_{2}, C_{2}\right)$. This transition is defined from a stochastic matrix on $\mathcal{M}$.

Figure 2 displays a possible path given by the model.

Figure 2 approximately here.

\section{Transition of FCG between region II and region III}

The aim is to investigate the transition between regions II and III of propagation. Region II of the crack propagation is assimilated to the Paris linear regime (see Figure 1). This is justified by the analysis of the crack growth rates in terms of $\Delta K$ (see Figure 3). This transition is assumed to occur at the regimeswitching time between Paris and Forman equations. We need a model that allows a regime-switching and PDMP's introduced in the previous section are particularly well-adapted for this purpose. Paris equation (1) is considered for the crack growth propagation $\mathcal{G}_{M_{1}}$ corresponding to region II and Forman law (3) is used for $\mathcal{G}_{M_{2}}$ corresponding to the rapid crack propagation in region III. $T$ is the random time of jump between both regimes. Experimental data provided in [1] are fitted to theoretical curves issued from the model and a set of parameters is obtained then statistically analysed.

\subsection{Experimental data}

In [1], 62 identical centre-cracked aluminium alloy specimens (152 $\mathrm{mm}$ wide and $2.54 \mathrm{~mm}$ thick) were tested under constant amplitude loading $\Delta \sigma=48.28 \mathrm{MPa}$ at a stress ratio $R=0.2$. The number of loading cycles for the crack tip to advance a predetermined increment $\Delta a$ was recorded from an initial crack length of 9 $\mathrm{mm}$ to a final length of $49.8 \mathrm{~mm}$. 68 crack growth histories were obtained from these tests. For each crack and each measurement $1 \leq i \leq 164, a^{i}$ denotes the crack length after $N^{i}$ loading cycles. These quantities represent the empirical data required to build the proposed modelling. The 68 experimental crack length curves versus the number of cycles are presented in Figure 3. A large scatter is obtained which corresponds to the variability of the crack growth process. The logarithmic representation of the crack growth rate in terms of $\Delta K$ is also presented in Figure 4. As it was schematized in Figure 1, a linear increase of $\mathrm{d} a / \mathrm{d} N$ is observed on the largest part of the propagation with some changes at the end and to a lesser extend, at the beginning.

Figures 3 and 4 approximately here. 


\section{$3.2 \quad$ Fit of experimental curves}

This section is devoted to the determination, for each experimental curve, of the nearest theoretical curve coming from the model. This problem is addressed with an optimisation formulation. By definition of our model, a theoretical curve is determined by five parameters $\left(m_{1}, C_{1}, T, m_{2}, C_{2}\right)$ with $\left(m_{1}, C_{1}\right)$ the Paris law parameters, $\left(m_{2}, C_{2}\right)$ the Forman law parameters and $T$ the jump time. Thus, to properly fit each experimental curve to a theoretical one, the optimal parameters $\left(m_{1}^{*}, C_{1}^{*}, T^{*}, m_{2}^{*}, C_{2}^{*}\right)$ that minimise an objective function must be determined. The latter measures the distance between crack lengths given by the experimental curve and by the theoretical one obtained by discretisation of deterministic laws. For each experimental curve, the optimisation problem can be stated as follows:

$$
\underset{\left(m_{1}, C_{1}, T, m_{2}, C_{2}\right)}{\operatorname{Minimise}} f\left(m_{1}, C_{1}, T, m_{2}, C_{2}\right)=\sum_{i=1}^{164}\left[a_{\text {theo }}^{i}\left(m_{1}, C_{1}, T, m_{2}, C_{2}\right)-a_{\exp }^{i}\right]^{2},
$$

where $a_{\text {theo }}^{i}$ and $a_{\exp }^{i}$ are theoretical and experimental crack lengths at each measurement $i$, respectively. The authors would like to emphasise that this optimisation problem is far to be obvious. Initially, the values assigned to the material parameters are randomly selected from the range of values, and a simulated annealing algorithm is used to solve the above optimisation problem. A metaheuristic algorithm is justified by its ability to determine the global optimum even in this highly non-convex context. The reader can find further information about the implementation of simulated annealing algorithms and parameter selection in $[38,39]$.

\subsection{Results}

The quality of the fitting is examined first. Figure 5 displays the graphs of the worst (left) and the best (right) fitted versions of the experimental curves among the 68's. In each case, the model fits very well the crack length evolution. It may be pointed out that the end of crack propagation is accurately described by the regime-switching model using Forman's law for the transition between region II and region III as shown in Figure 6 that focuses on the end of propagation of three experimental curves fitted by either the regime-switching model, or another one with the Paris law for the second regime. A connection can be established between this result and the comment of [27] already presented in Subsection 2.3. Actually the authors of [27] notice that, in their model, the number of jumps increases at the end of the propagation. According to us, this result is due to the rapid increase of the crack propagation related to the beginning of crack growth instability which is characterized, in our case, by the switching to a more appropriate law.

\begin{tabular}{ccccc} 
& Mean & Standard deviation & Minimum & Maximum \\
\hline$m_{1}$ & 2.8362 & 0.1421 & 2.3291 & 3.2605 \\
$\log \left(C_{1}\right)$ & -25.9688 & 0.8405 & -28.5290 & -23.0989 \\
$m_{2}$ & 3.6354 & 0.1233 & 3.2448 & 3.9218 \\
$\log \left(C_{2}\right)$ & -25.3412 & 1.0853 & -25.7594 & -22.2450 \\
\hline
\end{tabular}

Table 1: Statistics of parameters $m$ and $\log (C)$ for the first and second regimes.

Figures 5 and 6 approximately here.

Statistics about $m$ and $\log (C)$ for each regime and relationships between them are presented in Table 1 and Figure 7. The values of the coefficient of multiple correlation $R^{2}$ for both Paris and Forman laws demonstrate the accuracy of the linear model between these parameters. This correlation between $m$ and $\log (C)$, already known in the literature for propagation with only one regime (see [40,41, 42]) is again observed in both regimes of our model. According to [42], the correlation between Paris parameters $m$ and 
$\log (C)$ is due to the fact that the transition from Paris' regime (region II) to region III coincides with the Griffith-Irwin theory of instability. On the other hand, the authors of [41] stated that the high correlation between $m$ and $\log (C)$ is only due to the logarithmic representation. It should be mentioned that, since the relationship between parameters is found for both regimes with Paris and Forman laws, the correlation is only formal without real physical relevance. However, this linear relationship between $m$ and $\log (C)$ in each region, very helpful for the to model of PDMP, will be used in Section 4.

Figure 7 approximately here.

From now on, we focus on the analysis of the transition time, that is the crack length, the number of cycles and the stress intensity factor range $\Delta K$ values at the estimated transition time $T^{*}$. Their statistical characteristics are listed in Table 2. As expected, the jump associated to the transition between Paris and Forman laws occurs at the end of the propagation. The mean value of $\Delta K$ is equal to $20.8 \mathrm{MPa} \sqrt{m}$ and corresponds to the end of the linear part of the $\log (\mathrm{d} a / \mathrm{d} N)$ versus $\log (\Delta K)$ curve (see Figure 4$)$ obtained for $\Delta K=21 \mathrm{MPa} \sqrt{m}$. Considering that the crack instability condition is reached for $K_{\max }=K_{C}$ at the transition between region II and region III, the corresponding stress intensity factor is given by:

$$
\Delta K=K_{\max }-K_{\min }=(1-R) K_{C},
$$

where $R=K_{\min } / K_{\max }=0.2$. Mean, maximal and minimal values of fracture thoughness $K_{C}$ connected with $\Delta K$ values obtained from the above equation are presented in Table $2 . K_{C}$ values are contained between 20 and $32 \mathrm{MPa} \sqrt{m}$. In handbooks [43, 44], minimal and maximal values for fracture thoughness determined experimentally for the 2024-T3 aluminium alloy are equal to $15 \mathrm{MPa} \sqrt{m}$ and $50 \mathrm{MPa} \sqrt{m}$, respectively. There is a good agreement between estimated values obtained in this study and lower values recorded in handbooks. This result means that the choice of PDMP with only one jump combined with appropriate deterministic laws gives results with significant physical relevance in terms of $\Delta K$. This quantity itself is deterministically related to the length of the cracks through equation (2). The knowledge of such a critical crack length is very important for safety of structures and may be used as a criterion for a designer to avoid sudden failures.

\begin{tabular}{ccccc} 
& Mean & Standard deviation & Minimum & Maximum \\
\hline Crack length $(\mathrm{mm})$ & 39.53 & 4.55 & 30.40 & 48.20 \\
Transition times $($ number of cycles) & 241401 & 19184 & 192389 & 296091 \\
$\Delta K(\mathrm{MPa} \sqrt{m})$ & 20.8 & 1.0544 & 16.5 & 25.3 \\
$K_{C}(\mathrm{MPa} \sqrt{m})$ from equation $(5)$ & 25.8 & NA & 20.6 & 32 \\
\hline
\end{tabular}

Table 2: Statistics about the crack length at the jump time, the transition time and the corresponding stress intensity factor range.

\section{Prediction of fatigue crack propagation}

The purpose of this part is to predict the whole trajectory of FCG on some device from the knowledge of a few inspections performed at the beginning of the propagation. It is assumed that the device is no more available for monitoring after a certain time. Nevertheless, the first measurements are used to better forecast the future crack path. The framework is thus simpler than real-time monitoring, which has been investigated by some authors like $[45,16,46,19]$. From a mathematical point of view, it consists in predicting the crack behaviour knowing only the first points of its curve. The principle of the updating method is the following: a suitable model of crack propagation is constructed and then, the knowledge of the beginning of propagation of a given crack allows us to reduce the possible paths inside the model, these paths leading to the future of this given crack. We present first the dynamic model and the updating method, then the quality of the prediction is analysed for the whole trajectory (phases II and III) of each crack of the Virkler dataset. 


\subsection{Calibration of the model}

We consider a PDMP model for crack propagation updated from measurements taken the beginning of the propagation. As a consequence, Forman's law, very suitable to detect crack growth exit of the linear regime of Paris, is not well-adapted in this new context. For this reason, we propose a model only based on the Paris' law both before and after the jump time at which only material parameters $m$ and $C$ change. In the framework of Subsection 2.3, we model crack propagation as follows: crack growth rate is first driven by Paris equation (1) with a set of parameters and at a random time, the set of parameters related to Paris dynamic changes. To avoid any confusion with the previous work presented in Subsection 2.3, this new model is called Paris model with one jump. Randomness comes again from three sources: the stochastic choice of parameters at the beginning denoted by $\left(m_{1}, C_{1}\right)$, the random jump time $T$ and the random transition between parameters $\left(m_{1}, C_{1}\right)$ and $\left(m_{2}, C_{2}\right)$. Nevertheless, we would like to emphasise that the aim of this second investigation is to provide a simulation model suitable to predict FCG, while the goal of the previous part was to capture the transition between regimes II and III of the propagation.

Let us recall that the features of a PDMP for crack propagation are the finite state space $\mathcal{M}$ for the material parameters $(m, C)$, their initial distribution, their transition matrix and finally the distribution of the jump time. The last three govern the randomness of our simulation model and will be estimated by their empirical version. First of all, we mimic Subsection 3.2 and more precisely equation (4) to fit each experimental curve to a theoretical one issued from the Paris model with one jump. These new data $\left(m_{1}^{*}, C_{1}^{*}, T^{*}, m_{2}^{*}, C_{2}^{*}\right)$ are used to fit the features of our simulation model:

- Finite state space $\mathcal{M}$ for the parameters $(m, C)$

We group the 68 values of $m_{1}^{*}$ in $p$ classes and we propose the centers of these classes as a first set of points for $m$. Then we use the linear link between $m$ and $\log (C)$ to propose two corresponding values of $C$ at each class center $m$, that is $\log (C)=a+b m+\sigma$ and $\log (C)=a+b m-\sigma$ where $a, b$ and $\sigma$ have been estimated from the linear regression between $m_{1}^{*}$ and $\log \left(C_{1}^{*}\right)$. Now we have a first set of $2 p$ pairs of $(m, C)$ corresponding to the first regime of prediction for the given crack. A second set of points for $\mathcal{M}$ is chosen using the fitted law of $\log \left(C_{1}^{*}\right)-\log \left(C_{2}^{*}\right)$ (Gaussian in our case) and again from the linear correlation between $m_{2}^{*}$ and $\log \left(C_{2}^{*}\right)$. The final set $\mathcal{M}$ thus contains $4 p$ pairs $(m, C)$.

- Initial distribution of parameters $(m, C)$

The initial law of these parameters is given by a probability law on $\mathcal{M}$ obtained as the empirical distribution of the 68 values of $m_{1}^{*}$ on the classes obtained at the previous step.

- Jump time distribution

The jump time law depends on the parameters $(m, C)$. Thus, we simply use an exponential variable with constant coefficient estimated by maximum likelihood in each mode of $(m, C)$.

- Transition distribution of parameters $(m, C)$

The transition law between parameters $\left(m_{1}, C_{1}\right)$ and $\left(m_{2}, C_{2}\right)$ is given by a transition matrix on $\mathcal{M}$ obtained from the empirical conditional distribution of the 68 values of $\left(m_{1}^{*}, C_{1}^{*}\right)$ and $\left(m_{2}^{*}, C_{2}^{*}\right)$ on the classes build at the first step.

Figure 8 shows a set of simulated curves from $p=1$. It should be noted that in this case there are only 4 possibles values for parameters $m$ and $C$ in $\mathcal{M}$. We see that even if we have two possible curves at the beginning of the propagation, and only two propagation regimes after the jump time $T$, the bundle is rich because of the diversity of jump times. We can note that the simulated bundle includes the experimental one (see Figure 3). It is also interesting to choose $p>1$ in the purpose of prediction. This is the object of the next section.

Figure 8 approximately here. 


\subsection{Updating method}

The idea developed in [47] and [28] is followed here. The assumption of the updating method is to use the information of the first points of the propagation of a given crack in order to reduce the number of possible trajectories of the model: it means that the set of parameters $(m, C)$ in $\mathcal{M}$ is reduced to predict the future of the propagation. It leads to a thin and precise bundle of crack paths. Suppose that $\ell$ measures from an experimental curve are observed. The Paris model contains $2 p$ theoretical curves corresponding to $2 p$ values of $(m, C)$ for the first part of the propagation. For a given experimental curve, from $\ell$ points of measure, the distance to each of the $2 p$ theoretical curves defined by the model for the beginning of the propagation can be defined as the cost function

$$
f(m, C)=\sum_{i=1}^{\ell}\left[a_{\text {theo }}^{i}(m, C)-a_{\text {exp }}^{i}\right]^{2} .
$$

We proceed in three steps:

Step 1. The $2 p$ values $f(m, C),(m, C) \in \mathcal{M}$, are computed in order to choose the $r(r<2 p)$ nearest curves among the $2 p$ theoretical curves of the model. Figure 9 (top) summarizes this procedure for $p=5$ and $r=4$ : the experimental measurements are drawn with black points and here the 4 nearest theoretical curves (solid lines) are chosen among the $2 p=10$ possible paths.

Step 2. We work with each of the $r$ possible trajectories chosen at the first step and corresponding to $r$ values of $(m, C)$. For a given crack and each number of cycles $N^{i}$ corresponding to the point of measure $a_{\text {exp }}^{i}$, the $2 p$ possible paths corresponding to the parameters $(m, C)$ of the second regime starting from the point $\left(N^{i}, a_{\text {theo }}^{i}(m, C)\right)$ are drawn. The distance to the second part of the experimental curve is computed. Again the $r$ nearest among the $2 p$ possible paths are chosen. This step is illustrated in Figure 9 (bottom) for the jump time at the third measure with $p=5$ and $r=4$.

Step 3. We are now ready for the simulation of a crack in the prediction bundle of the experimental curve. A curve is randomly chosen in the $r$ theoretical curves defined at step 1 according to the law of the initial distribution of $(m, C)$ restricted to the $r$ curves. The jump time is drawn according to the jump distribution determined for this curve. If this jump occurs before the last observation, we choose it, otherwise, the jump time is made at the $(\ell-1)^{\text {th }}$ observation. For the second part of the propagation, we choose randomly between the $r$ theoretical curves corresponding to this jump time and determined at step 2. The law is the transition distribution given by the model and restricted to the $r$ curves. We repeat this scheme for each simulated FCG to build the prediction bundle.

Figure 9 approximately here.

\subsection{Validation and results}

Unlike [28] in which the authors performed their simulation study only on some specific cracks and until 250000 cycles, the whole trajectory is predicted for all cracks. A leave-one-out method is proposed in order to tackle the versatility of our updating procedure. Indeed, for each experimental crack, the parameters of the Paris model are computed with the 67 remaining cracks. From the $\ell$ first points of propagation of the involved crack, the obtained model is used to simulate a prediction bundle until the final length $49.8 \mathrm{~mm}$ (corresponding to a final number of cycles between 222000 and 321000 cycles according to the crack) and the quality of the prediction is investigated with the following criterion: the distance between the crack and its prediction bundle. This quantity equals 0 if and only if the crack remains inside the bundle along the propagation. More precisely, we determine for each measurement $a_{\exp }^{i}, \ell \leq i \leq 164$, the simulated curve of the prediction bundle that reaches first the size $a_{\exp }^{i}$, and $\nu_{\min }^{i}$ denotes the corresponding number of cycles. The curve that reaches this size at last is also computed and $\nu_{\max }^{i}$ stands for the corresponding number of 
cycles. The quantity $d^{i}$ denotes the distance between the experimental curve and its prediction bundle at measure $i$ and is defined by:

$$
\begin{array}{lll}
d^{i}=0 & \text { if } & \nu_{\min }^{i} \leq N^{i} \leq \nu_{\max }^{i} \\
d^{i}=\nu_{\min }^{i}-N^{i} & \text { if } & N^{i} \leq \nu_{\min }^{i} \\
d^{i}=N^{i}-\nu_{\max }^{i} & \text { if } \quad \nu_{\max }^{i} \leq N^{i}
\end{array}
$$

It should be underlined that the couples $\left(\nu_{\min }^{i}, a_{\exp }^{i}\right)$ and $\left(\nu_{\max }^{i}, a_{\exp }^{i}\right)$ define the extreme curves of the prediction bundle. The overall distance between the experimental curve and its prediction bundle is the sum of these local distances normalised by the total number of cycles $N^{164}$ used to reach the final length 49.8 $\mathrm{mm}$, that is:

$$
D=\frac{1}{N^{164}} \sum_{i=\ell+1}^{164} d^{i} .
$$

Results of the leave-one-out method using the model with $p=5$, that is to say from 20 possible sets of parameters, have been selected. Three different crack behaviours are distinguished according to the number of cycles reached after 160 measurements (end of propagation). Cracks are considered rapid for $N^{160}<240000$ cycles, while they are slow for $N^{160}>280000$ cycles, and thus average for $240000<N^{160}<280000$ cycles. Figure 10 presents three experimental curves with their predicted bundle for two rapid cracks and a slow one. The normalised distance values $D$ defined earlier are calculated for both cracks. For the rapid crack, the experimental curve is always located in the bundle $(D=0)$ or goes out only during a short time around 125000 cycles $(D \simeq 0.2)$. For the slow crack, the experimental curve, is not well-predicted and the corresponding value of $D$ is about 11.6. In this case, the real crack path is discountinuous and presents irregularities due to local microstructural variations characterised by the stop or the slowing down of the crack. However, in this case, the bundle is slightly situated above the experimental curve showing that, in terms of prediction, the model overestimates the real crack behaviour. Overall results of the cross validation are presented in Table 3. The quantity $d^{160}$ indicates if the crack is in the bundle at the end of its propagation. $38 \%$ of the experimental curves remain inside the bundle along the propagation $(D=0)$, $72 \%$ are at a very low distance $(0 \leq D<1)$, and two among three are inside the bundle at the end of the propagation $\left(d^{160}=0\right)$. Furthermore, it should be noted that all the rapid cracks are well-forecasted $(0<D<1)$, while any slow crack is accurately simulated $(D>1)$. For slow and some average cracks, the path is not always well-predicted but, in these cases, the bundle is always located above the experimental curve. Consequently, this kind of method does not always accurately describe the whole crack path, in particular in the difficult framework of slow cracks as it is the case in Figure 10 (bottom). Actually, the model always overestimates irregular slow cracks, which is a good point to systematically reduce the risk of rupture, probably because of the micro-inhomogeneities along the crack path. On the contrary, the prediction carried out for rapid cracks and for a large number of average cracks, which appear as the most dangerous situations, is very powerful.

Figure 10 approximately here.

\begin{tabular}{cccc} 
& Rapid cracks & Average cracks & Slow cracks \\
Occurrences & 11 & 50 & 7 \\
\hline$D=0$ & 8 & 18 & 0 \\
$0<D<1$ & 3 & 20 & 0 \\
$D>1$ & 0 & 12 & 7 \\
$d^{160}=0$ & 9 & 35 & 1 \\
\hline
\end{tabular}

Table 3: Normalised distance $D$ and crack location at the end of the propagation $d^{160}$ for the different types of crack. 


\section{Conclusion}

The ability of a simple model of PDMP to address specific problems of fatigue crack propagation has been highlighted in this work. Virkler data have been used in order to confirm the efficiency of our model. In the first part of the paper, we have investigated the advantage to use a switching model with two adapted deterministic laws to capture the change between region II and region III where the crack growth instability occurs. It has been emphasised that the linear relationship between the material parameters $m$ and $\log (C)$ exists in both regimes of propagation. The transition to region III has been detected and and described in terms of number of cycles, length and stress intensity factor. In addition, the mean stress intensity factor range resulting from the model is very close to the minimal fracture thoughness proposed in handbooks for the 2024-T3 aluminium alloy. The idea developed in this paper could be extended to the modelling of the transition between region I and region II by using specific deterministic propagation laws for small cracks. The second problem investigated in this article deals with the crack path prediction using few information at the beginning of the propagation. In practice, the length evolution of a crack found in structural components subjected to cyclic loading can be obtained when structures are periodically inspected with non-destructive technics (NDT). It has been highlighted that the PDMP model combined with an efficient updating method is able to predict the FCG until the rapid crack propagation regime. According to [48] results provided that a good inspection at the beginning of the propagation is very useful to predict the whole propagation and particularly the time to reach a critical length. It could be used to plan further inspections. A dynamic model is well-adapted probably because it takes into account the transition to region III. The proposed study has stated the potential of the PDMP model to predict crack propagation based on the knowledge of some information that may be issued from NDT. In particular, this may be applied to safety monitoring of structures after intensive tests on other datasets (for instance simulated via NASGRO software [20]) and of course on real structures for validation. There are other perspectives like the estimation of the remaining life, online updating of the prediction from new measurements and the use of PDMP's for modelling crack propagation in other materials (probably with different deterministic laws).

\section{Acknowledgements}

This work was supported by ARPEGE program of the French National Agency of Research (ANR), project "FAUTOCOES" number ANR-09-SEGI-004.

\section{References}

[1] Virkler D, Hillberry B, Goel P. The statistical nature of fatigue crack propagation. J Engng Mater Technol. 1979;101(2):148 - 153.

[2] Wu WF, Ni CC. Statistical aspects of some fatigue crack growth data. Engineering Fracture Mechanics. 2007;74(18):2952 - 2963. Reliability - Statistical Methods in Fracture and Fatigue.

[3] Casciati F, Colombi P, Faravelli L. Inherent variability of an experimental crack growth curve. Structural Safety. 2007;29(1):66 - 76 .

[4] Moreno B, Zapatero J, Domínguez J. An experimental analysis of fatigue crack growth under random loading. International Journal of Fatigue. 2003;25(7):597 - 608 .

[5] Ghonem H, Dore S. Experimental study of the constant-probability crack growth curves under constant amplitude loading. Engineering Fracture Mechanics. 1987;27(1):1 - 25.

[6] Righiniotis TD, Chryssanthopoulos MK. Probabilistic fatigue analysis under constant amplitude loading. Journal of Constructional Steel Research. 2003;59(7):867 - 886. 
[7] Mohanty JR, Verma BB, Ray PK. Prediction of fatigue crack growth and residual life using an exponential model: Part I (constant amplitude loading). International Journal of Fatigue. 2009;31(3):418 424.

[8] Sankararaman S, Ling Y, Mahadevan S. Uncertainty quantification and model validation of fatigue crack growth prediction. Engineering Fracture Mechanics. 2011;78(7):1487 - 1504.

[9] Chang JB, Hudson CM, on Fracture Mechanics Applications ASE. Methods and Models for Predicting Fatigue Crack Growth Under Random Loading. A.S.T.M. STP. ASTM; 1981.

[10] Xiang Y, Liu Y. Application of inverse first-order reliability method for probabilistic fatigue life prediction. Probabilistic Engineering Mechanics. 2011;26(2):148 - 156.

[11] Zapatero J, Domínguez J. A statistical approach to fatigue life predictions under random loading. International Journal of Fatigue. 1990;12:107 - 114.

[12] McMaster FJ, Smith DJ. Predictions of fatigue crack growth in aluminium alloy 2024-T351 using constraint factors. International Journal of Fatigue. 2001;23:93 - 101.

[13] Wu WF, Ni CC. A study of stochastic fatigue crack growth modeling through experimental data. Probabilistic Engineering Mechanics. 2003;18:107 - 118.

[14] Paris PC, Erdogan F. A Critical Analysis of Crack Propagation Laws. J Basic Engng. 1963;85(4):528 $-533$.

[15] Forman R, Keary V, Eagle R. Numerical Analysis of Crack Propagation in Cyclic-Loaded Structures. Trans ASME J Basic Engng. 1967;89(3):459 - 464.

[16] Cadini F, Zio E, Avram D. Monte Carlo-based filtering for fatigue crack growth estimation. Probabilistic Engineering Mechanics. 2009;24(3):367-373.

[17] Sobczyk K, Spencer Jr BF. Introduction: From Data to Theory. In: Spencer KSF, editor. Random Fatigue. San Diego: Academic Press; 1992. p. $1-7$.

[18] Yang JN, Manning SD. A simple second order approximation for stochastic crack growth analysis. Engineering Fracture Mechanics. 1996;53(5):677-686.

[19] Corbetta M, Sbarufatti C, Manes A, Giglio M. Sequential Monte Carlo sampling for crack growth prediction providing for several uncertainties. In: Proceedings of the 2nd European conference of the prognostics and health management society; 2014. .

[20] NASA. Fatigue crack growth computer program NASGRO Version 3.0-Reference manual. JSC-22267B, NASA, Lyndon B. Johnson Space Center, Texas; 2000.

[21] Skorupa M, Machniewicz T, Schijve J, Skorupa A. Application of the strip-yield model from the NASGRO software to predict fatigue crack growth in aluminium alloys under constant and variable amplitude loading. Engineering Fracture Mechanics. 2007;74(3):291 - 313.

[22] Beck AT, de Santana Gomes WJ. Stochastic fracture mechanics using polynomial chaos. Probabilistic Engineering Mechanics. 2013;34:26-39.

[23] Mattrand C, Bourinet J. Random load sequences and stochastic crack growth based on measured load data. Engineering Fracture Mechanics. 2011;78(17):3030-3048.

[24] Dhondt G. Application of the discrete Markov method to crack propagation problems. Int J Engng Sci. 1995;33(4):457 - 467 . 
[25] Zou XL. Statistical moments of fatigue crack growth under random loading. Theoretical and Applied Fracture Mechanics. 2003;39(1):1-5.

[26] Davis MHA. Piecewise-Deterministic Markov-Processes - A General-Class of Non-Diffusion StochasticModels. Journal Of The Royal Statistical Society Series B-Methodological. 1984;46(3):353-388.

[27] Chiquet J, Limnios N, Eid M. Piecewise deterministic Markov processes applied to fatigue crack growth modelling. Journal of Statistical Planning and Inference. 2009;139(5):1657 - 1667.

[28] Guan X, Giffin A, Jha R, Liu Y. Maximum relative entropy-based probabilistic inference in fatigue crack damage prognostics. Probabilistic Engineering Mechanics. 2012;29:157 - 166.

[29] Dufour F, Dutuit Y. Dynamic Reliability: A new model. In: Proceedings of $\lambda \mu 13$-ESREL02, Lyon, France; 2002. .

[30] Zhang H, Innal F, Dufour F, Dutuit Y. Piecewise Deterministic Markov Processes based approach applied to an offshore oil production system. Rel Eng \& Sys Safety. 2014;126:126 - 134.

[31] de Saporta B, Dufour F, Zhang H, Elegbede C. Optimal stopping for the predictive maintenance of a structure subject to corrosion. Journal of Risk and Reliability. 2012;226 (2):169-181.

[32] Beil M, Luck S, Fleischer F, Portet S, Arendt W, Schmidt V. Simulating the formation of keratin filament networks by a piecewise-deterministic Markov process. J Theo Biology. 2009;256:518-32.

[33] Davis MHA. Markov models and optimization. vol. 49 of Monographs on Statistics and Applied Probability. London: Chapman \& Hall; 1993.

[34] de Saporta B, Dufour F. Numerical method for impulse control of piecewise deterministic Markov processes. Automatica. 2012;48(5):779-793.

[35] de Saporta B, Dufour F, Gonzalez K. Numerical method for optimal stopping of piecewise deterministic Markov processes. Ann Appl Probab. 2010 10;20(5):1607-1637.

[36] Brandejsky A, de Saporta B, Dufour F. Optimal stopping for partially observed piecewise-deterministic Markov processes. Stochastic Processes and their Applications. 2013;123(8):3201-3238.

[37] Baysse C, Bihannic D, Gégout-Petit A, Prenat M, De Saporta B, Saracco J. Maintenance Optimisation of Optronic Equipment. In: Prognostics and System Health Management Conference; 2013. p. 709-714.

[38] Fabian V. Simulated annealing simulated. Computers \& Mathematics with Applications. 1997;33(1$2): 81-94$.

[39] Dréo J. Metaheuristics for Hard Optimization: Methods and Case Studies. Springer; 2006.

[40] Cortie M. The irrepressible relationship between the Paris law parameters. Engineering Fracture Mechanics. 1991;40(3):681 - 682 .

[41] Bergner F, Zouhar G. A new approach to the correlation between the coefficient and the exponent in the power law equation of fatigue crack growth. International Journal of Fatigue. 2000;22:229-39.

[42] Carpinteri A, Paggi M. Are the Paris' law parameters dependent on each other? Frattura ed Integrita Strutturale. 2007;2:10-16.

[43] ASM International. Properties and Selection: Nonferrous Alloys and Special-purpose Materials. ASM Handbook. ASM International; 1990.

[44] ASM International. Fatigue and fracture. vol. 19 of ASM Handbook. ASM International; 1996. 
[45] Corbetta M, Sbarufatti C, Manes A, Giglio M. On Dynamic State-Space models for fatigue-induced structural degradation. International Journal of Fatigue. 2014;61:202-219.

[46] Corbetta M, Sbarufatti C, Manes A, Giglio M. Real-Time Prognosis of Crack Growth Evolution Using Sequential Monte Carlo Methods and Statistical Model Parameters. Reliability, IEEE Transactions on. $2015 ; 64(2): 736-753$.

[47] Perrin F. Prise en compte des données expérimentales dans les modèles probabilistes pour la prévision de la durée de vie des structures; 2008. PhD dissertation, Université Blaise Pascal Clermont-Ferrand II, France.

[48] Ortiz K, Kiremidjian AS. A Stochastic Model for Fatigue Crack Growth Rate Data. Journal of Engineering for Industry, Transactions of the ASME. 1987;109:13-18. 


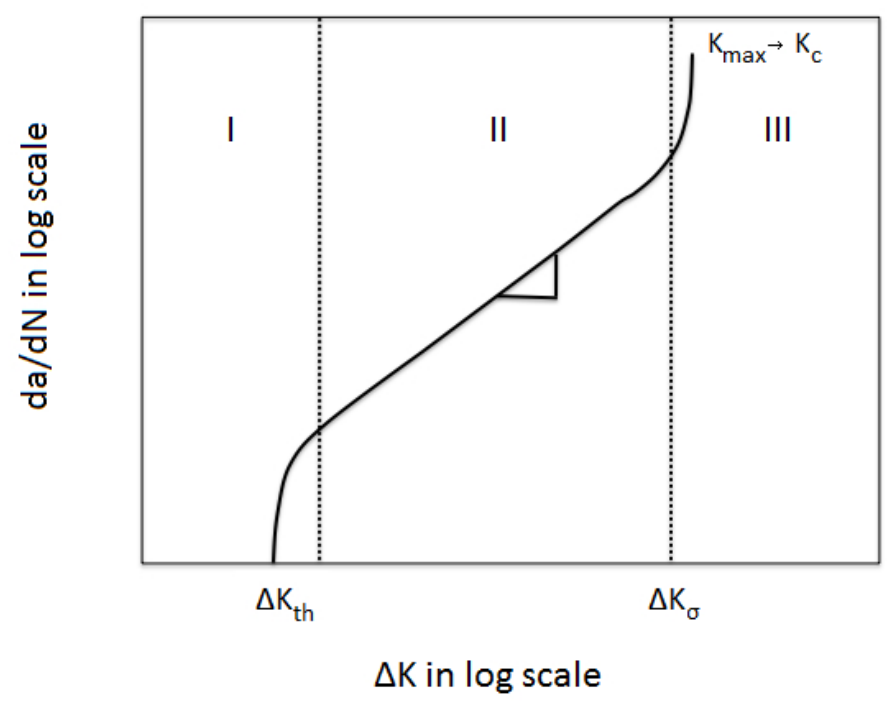

Figure 1: Schematic illustration of the different regimes of fatigue crack propagation. The vertical dashed lines indicate the transition between crack propagation regimes.

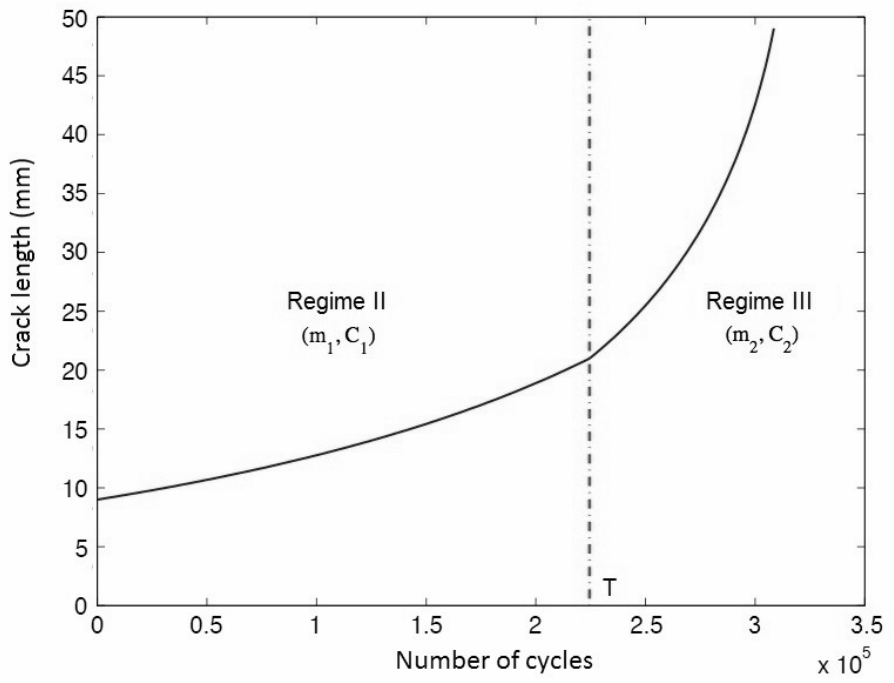

Figure 2: Example of a theoretical curve of the model of propagation. 


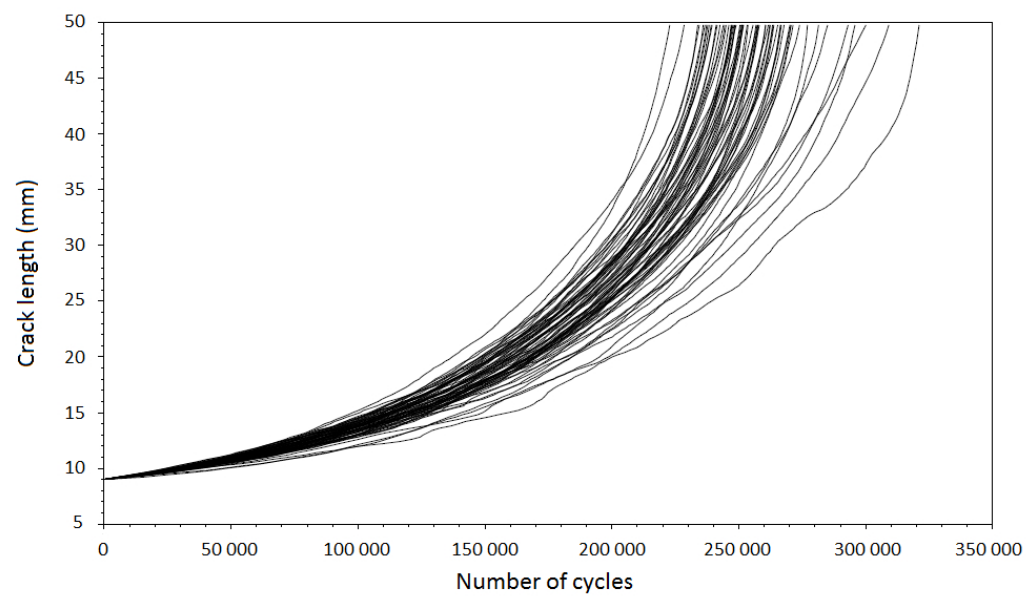

Figure 3: Crack length versus number of cycles for the 68 experiments provided in [1].

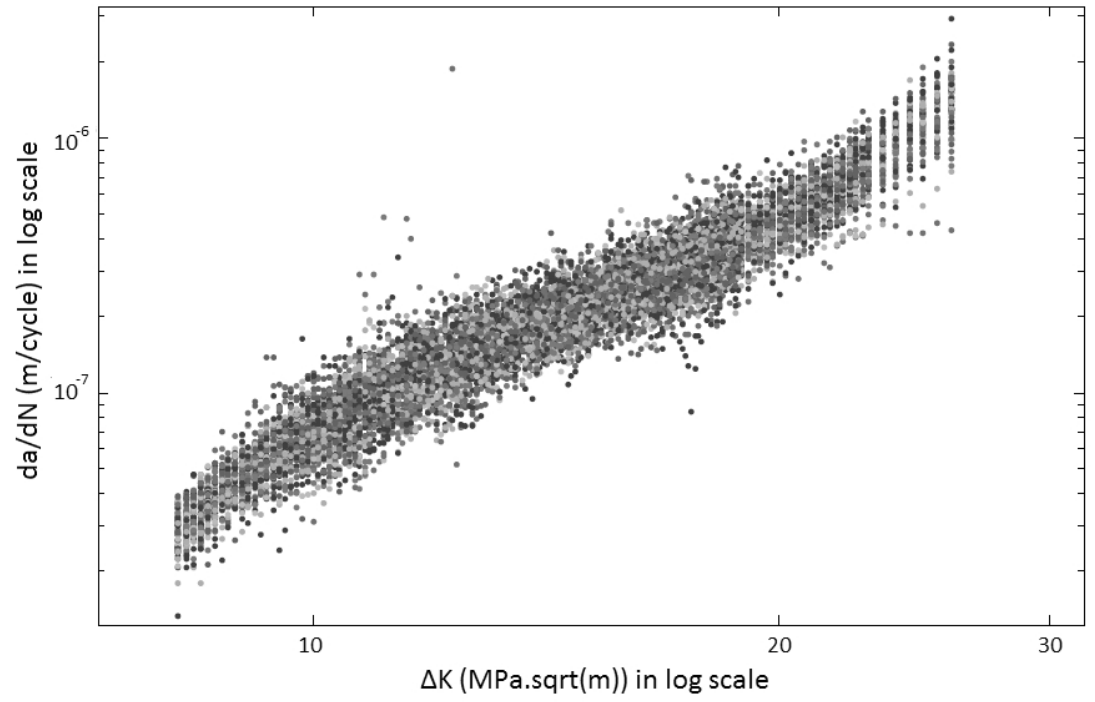

Figure 4: Crack growth rate in terms of $\Delta K$ computed from the 68 experiments provided in [1]. 

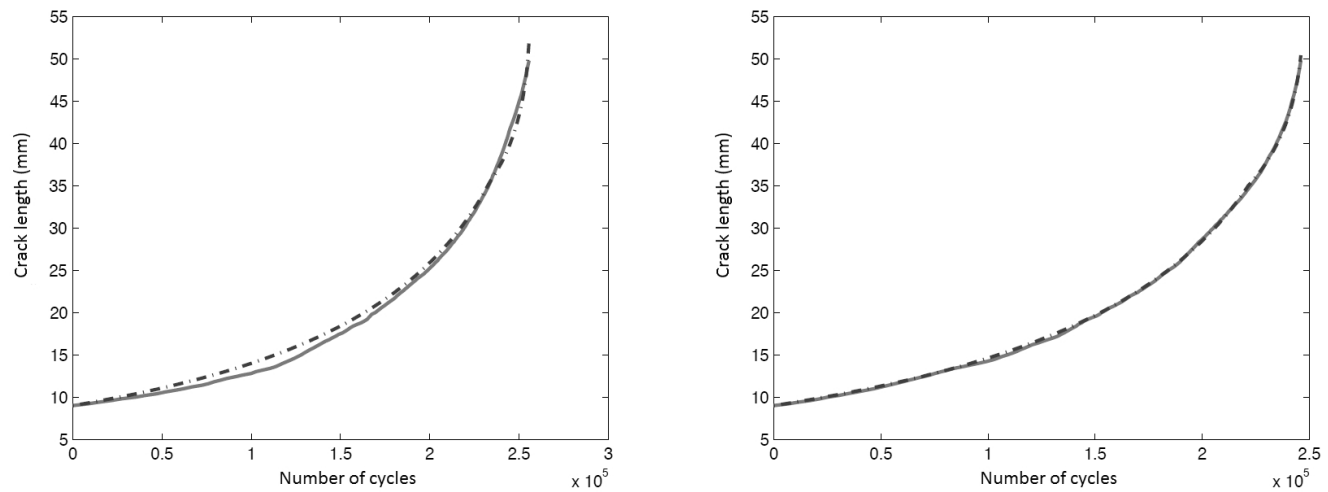

Figure 5: Experimental (solid line) and theoretical (dashed line) curves for the worst (left) and the best (right) fitted propagation length curves.
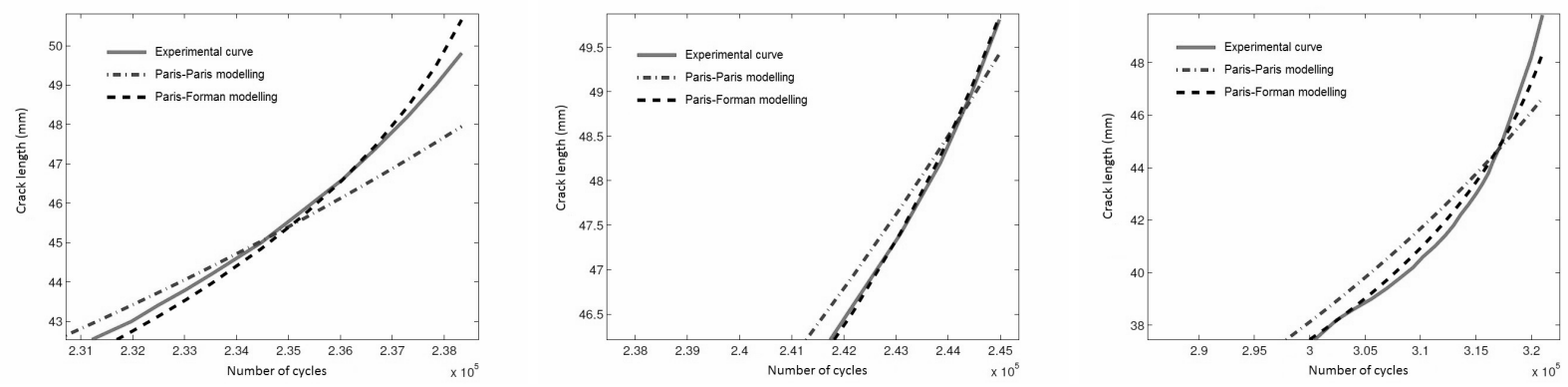

Figure 6: End of propagation for three different experimental cracks and fitting by regime-switching models with Paris or Forman law for the second regime. 

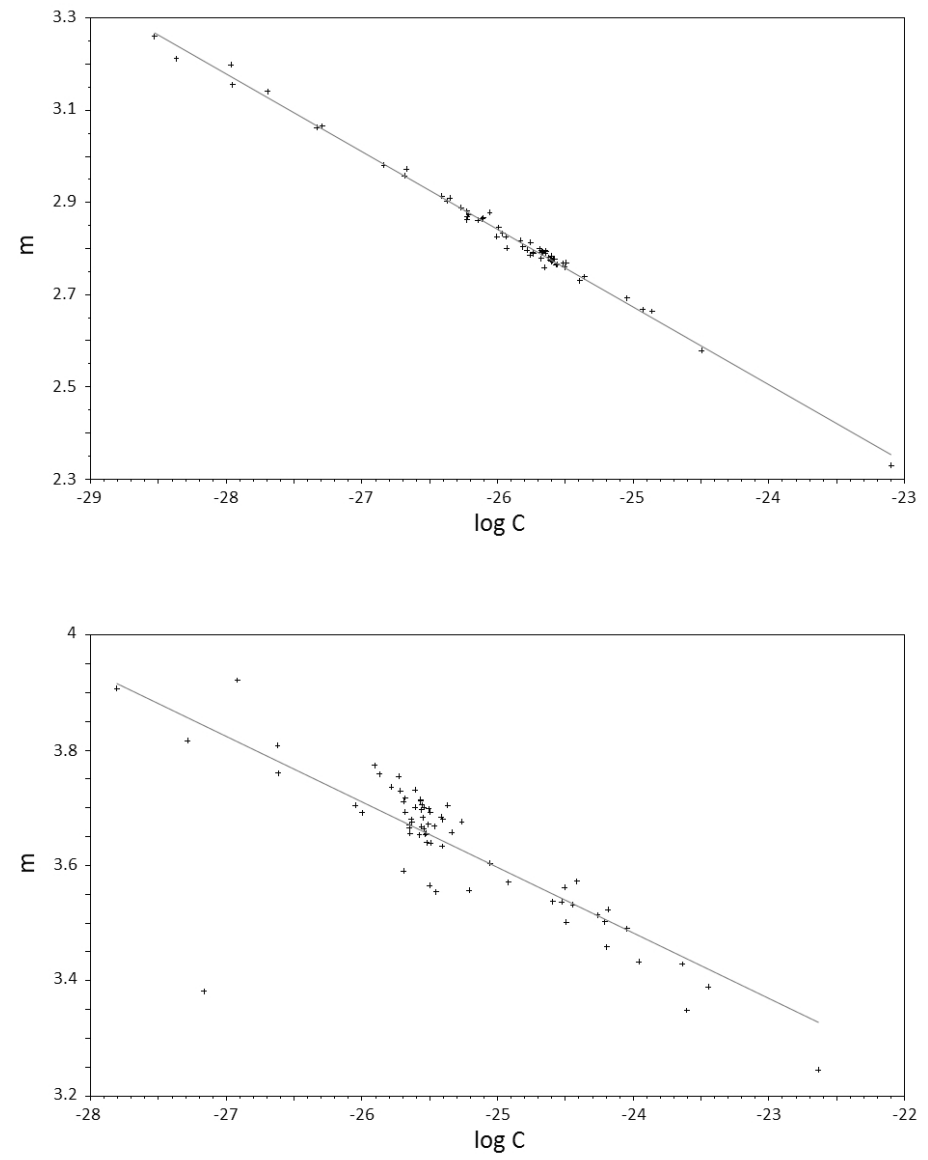

Figure 7: Linear relationship between the material parameters $m$ and $\log (C)$ in the Paris-Forman fitting in the first (top, $R^{2}=0.997$ ) and second (bottom, $R^{2}=0.819$ ) regimes. 


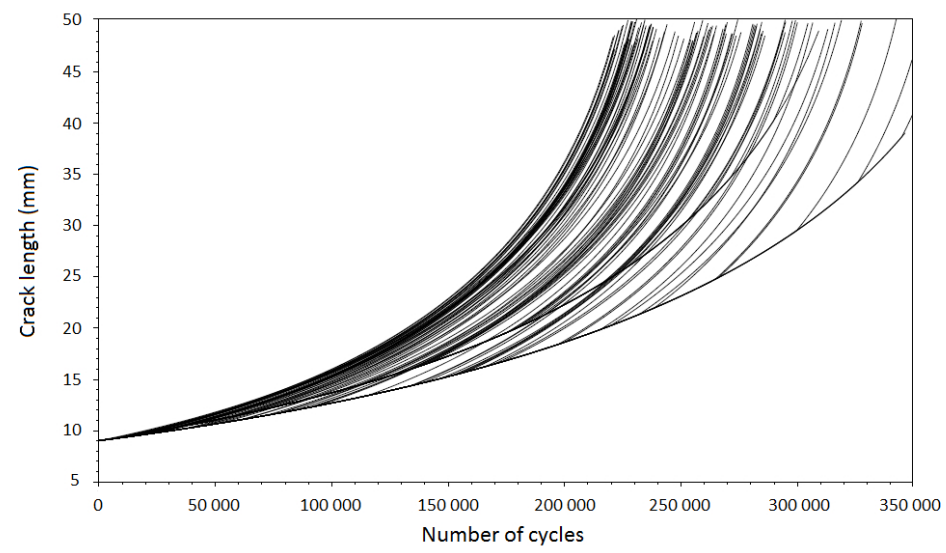

Figure 8: Simulation of crack propagation curves from parameter $p=1$. The dispersions of the experimental dataset and of the simulated bundle are quite similar. Indeed, all the experimental curves reach the final length between 210000 and 320000 cycles. This is also the case for more than $95 \%$ of the simulated curves presented in Figure 6. Note that there is only two curves of propagation before (resp. after) the transition. However the scattering of the bundle is due to the one of the jump times visible here. 

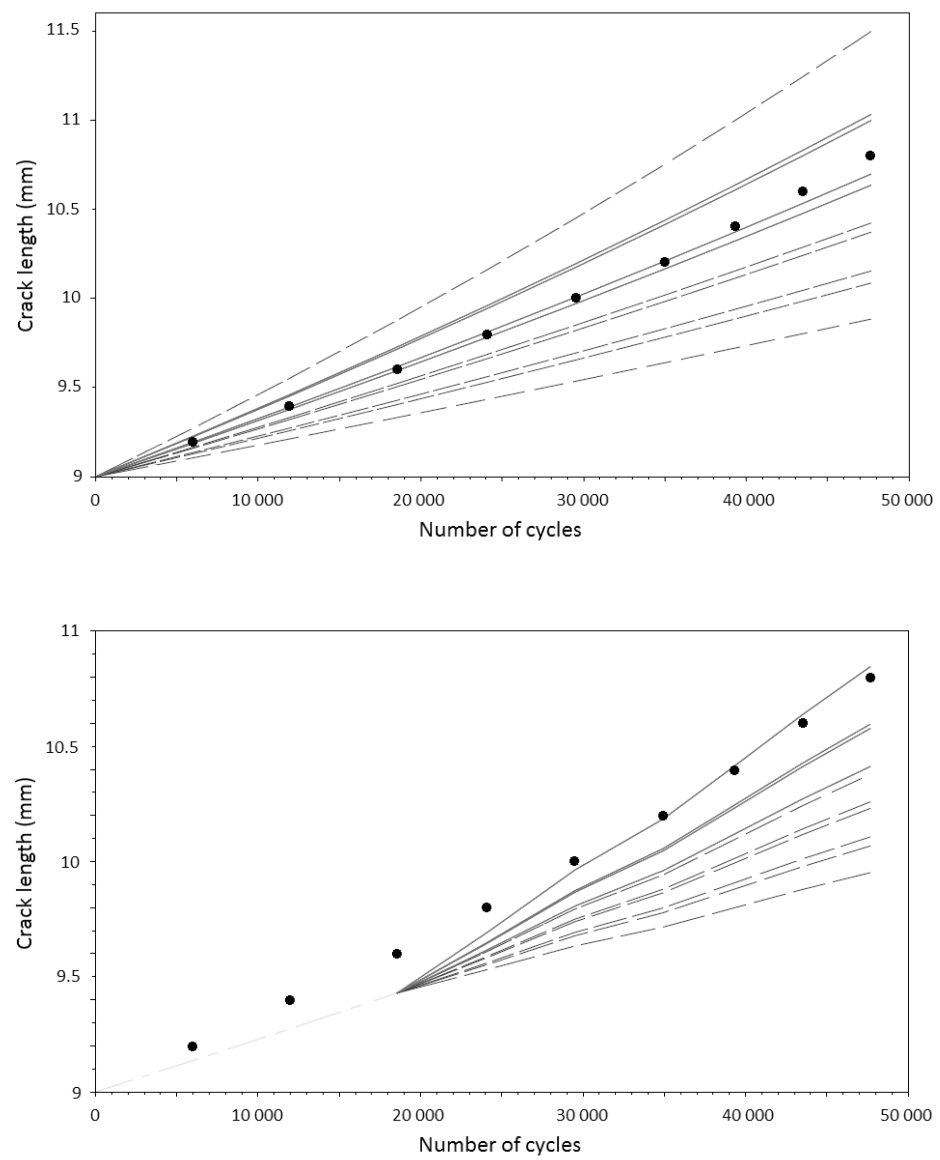

Figure 9: First (top) and second (bottom) steps of the updating method. Experimental measurements $(\ell=10)$ are drawn with black points and solid lines indicate the $r=4$ nearest theoretical curves among the $2 p=10$ possible paths. 

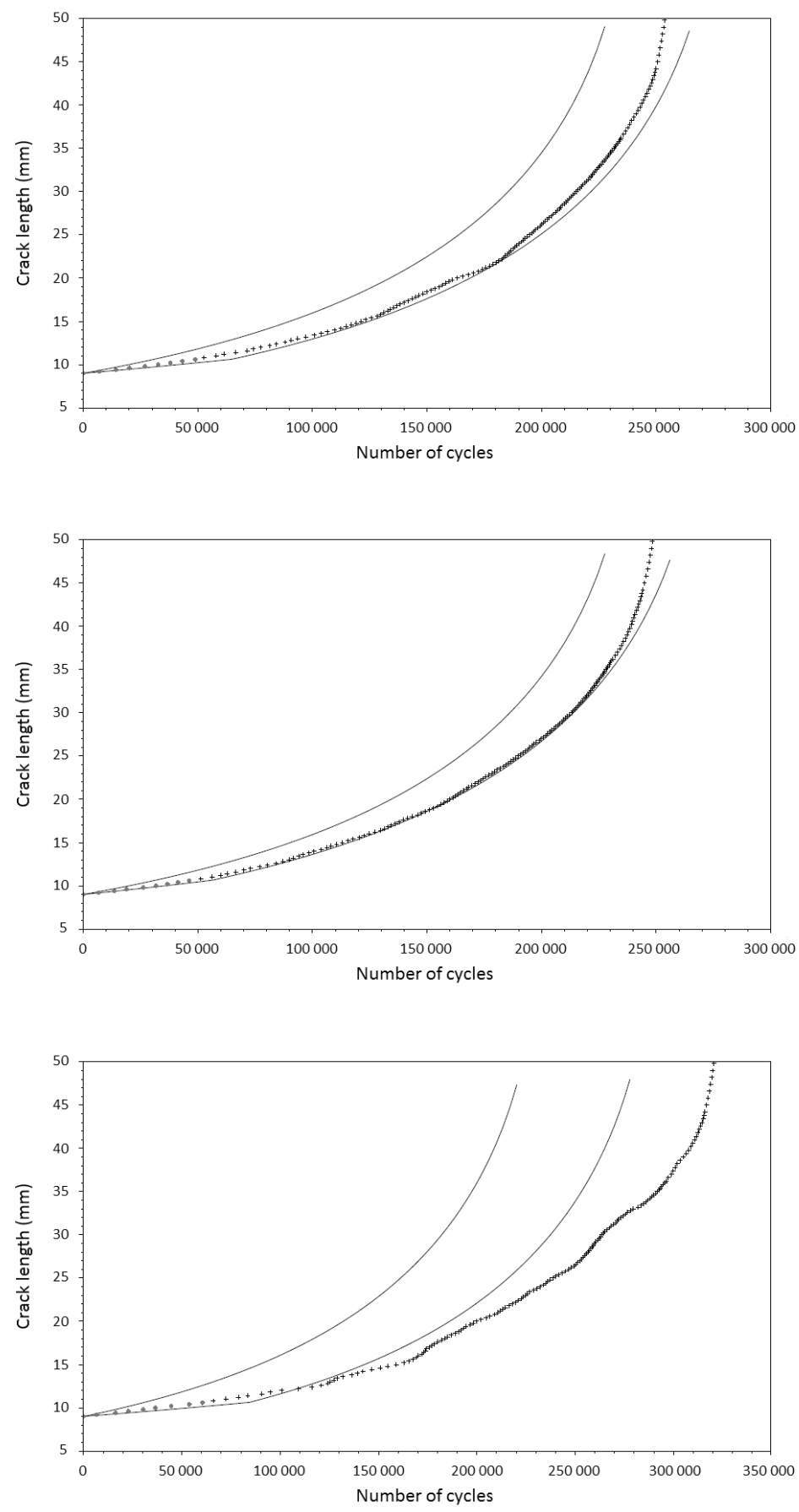

Figure 10: Three experimental propagations with the extreme curves of their prediction bundle obtained from the $\ell=10$ first measures: rapid cracks with $D=0$ and $D=0.2$ and slow crack with $D=11.6$ (from top to bottom). 